\title{
LOFAR early-time search for coherent radio emission from GRB 180706A
}

\author{
A. Rowlinson ${ }^{1,2 \star}$, K. Gourdji ${ }^{1}$, K. van der Meulen ${ }^{1}$, Z.S. Meyers ${ }^{1}$, T.W. Shimwell ${ }^{2}$, \\ S. ter Veen ${ }^{2}$, R.A.M.J. Wijers ${ }^{1}$, M.J. Kuiack ${ }^{1}$, A. Shulevski ${ }^{1}$, J.W. Broderick ${ }^{3}$, \\ A.J. van der Horst ${ }^{4,5}$, C. Tasse ${ }^{6,7}$, M.J. Hardcastle ${ }^{8}$, A.P. Mechev ${ }^{9}$, W.L. Williams ${ }^{8}$ \\ 1 Anton Pannekoek Institute, University of Amsterdam, Postbus 94249, 1090 GE Amsterdam, The Netherlands \\ 2 ASTRON, the Netherlands Institute for Radio Astronomy, Postbus 2, NL-7990 AA Dwingeloo, the Netherlands \\ 3 International Centre for Radio Astronomy Research, Curtin University, GPO Box U1987, Perth, WA 6845, Australia \\ 4 Department of Physics, the George Washington University, 725 21st Street NW, Washington, DC 20052, USA \\ 5 Astronomy, Physics and Statistics Institute of Sciences (APSIS), 725 21st Street NW, Washington, DC 20052, USA \\ 6 GEPI, Observatoire de Paris, Universite PSL, CNRS, 5 Place Jules Janssen, 92190 Meudon, France \\ 7 Department of Physics \& Electronics, Rhodes University, PO Box 94, Grahamstown, 6140, South Africa \\ 8 Centre for Astrophysics Research, School of Physics, Astronomy and Mathematics, University of Hertfordshire, \\ College Lane, Hatfield AL10 9AB, UK \\ 9 Leiden Observatory, Leiden University, PO Box 9513, NL-2300 RA Leiden, The Netherlands
}

Accepted XXX. Received YYY; in original form ZZZ

\begin{abstract}
The nature of the central engines of gamma-ray bursts (GRBs) and the composition of their relativistic jets are still under debate. If the jets are Poynting flux dominated rather than baryon dominated, a coherent radio flare from magnetic re-connection events might be expected with the prompt gamma-ray emission. There are two competing models for the central engines of GRBs; a black hole or a newly formed milli-second magnetar. If the central engine is a magnetar it is predicted to produce coherent radio emission as persistent or flaring activity. In this paper, we present the deepest limits to date for this emission following LOFAR rapid response observations of GRB 180706A. No emission is detected to a $3 \sigma$ limit of $1.7 \mathrm{mJy}^{\text {beam }}{ }^{-1}$ at $144 \mathrm{MHz}$ in a two-hour LOFAR observation starting 4.5 minutes after the gamma-ray trigger. A forced source extraction at the position of GRB 180706A provides a marginally positive (1 sigma) peak flux density of $1.1 \pm 0.9 \mathrm{mJy}$. The data were time-sliced into different sets of snapshot durations to search for FRB like emission. No short duration emission was detected at the location of the GRB. We compare these results to theoretical models and discuss the implications of a non-detection.
\end{abstract}

Key words: gamma-ray burst: individual: GRB 180607A - radio continuum: transients

\section{INTRODUCTION}

Since the first detection of gamma-ray bursts (GRBs), the community has been steadily gaining understanding of these events and their progenitor systems. Long GRBs are associated with core collapse supernovae (e.g. Hjorth et al. 2003; Woosley \& Bloom 2006) and short GRBs occur following the merger of two neutron stars (confirmed by the detection of GW 170817 and its association with GRB 170817A; Abbott et al. 2017) or a neutron star and a black hole. We now know that the prompt gamma-ray emission from GRBs

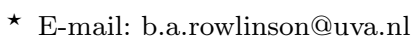

can be accompanied by TeV gamma-ray emission (Mirzoyan et al. 2019), X-rays and optical flashes. Astronomers have also searched for prompt radio emission, that could be associated with the central engine or the relativistic jet, but with no detections to date (Cortiglioni et al. 1981; Inzani et al. 1982; Koranyi et al. 1995; Dessenne et al. 1996; Balsano et al. 1998). These non detections are likely due to the small sample sizes of these studies (not all GRBs are likely to produce detectable radio emission in the same way as not all GRBs produce optical flashes) and relatively insensitive searches, typically $\gtrsim 100 \mathrm{Jy}$. One survey found a tantalising hint of two Fast Radio Bursts (FRBs), though at very low significance, associated with the X-ray plateau phases of 
two long GRBs (Bannister et al. 2012) but this has yet to be confirmed. With the exception of the work by Bannister et al. (2012), which used rapid response observations by the Parkes Radio Telescope, the previous surveys have typically been either whole sky instruments (with limited sensitivity) or hampered by very slow slew times. Recently, astronomers used the Murchison Widefield Array (MWA; Tingay et al. 2013), a low frequency radio telescope array with no moving parts, to enable a very rapid response observation of a short GRB (Kaplan et al. 2015) reaching a sensitivity of $\sim 1 \mathrm{Jy}$ on 30 minute time scales. Additionally, the Long Wavelength Array (LWA; Taylor et al. 2012), a whole sky transient survey instrument, was able to constrain prompt emission from a short GRB to a $1 \sigma$ flux density limit of $4.5 \mathrm{Jy} \mathrm{beam}^{-1}$ (Anderson et al. 2018).

An origin of prompt coherent radio emission from GRB events could be from magnetic re-connection events within a Poynting flux dominated jet (e.g. Drenkhahn \& Spruit 2002). The structure of the relativistic jets causing GRBs are still subject to investigation with Poynting flux dominated or baryon dominated jets being favoured (e.g. Sironi et al. 2015). We have observed coherent radio emission from magnetic re-connection events in the Sun, leading to a good understanding of the plasma physics involved (e.g. Bastian et al. 1998). Therefore, limits on coherent radio emission associated with the prompt emission can be used to constrain the presence of magnetic re-connection events (e.g. Inzani et al. 1982). One such model has been proposed by Usov \& Katz (2000), who consider the coherent radio emission expected from relativistic, strongly magnetised winds produced by GRBs.

Additionally, the nature of the central engine powering these GRBs is a subject of continuing debate with two key theories proposed: a black hole (e.g. Woosley 1993) or a millisecond spin period, highly magnetised, massive neutron star (hereafter referred to as a magnetar; e.g. Metzger et al. 2011). An observable signature of the magnetar model is a prolonged X-ray plateau phase (Zhang \& Mészáros 2001). As accretion ends within seconds for short GRBs, the plateau phases observed are typically associated with the magnetar model (e.g. Rowlinson et al. 2013, and references therein). However, for long GRBs, this plateau phase has been both associated with the magnetar central engine model (e.g. Bernardini et al. 2012) and with ongoing accretion onto the central engine (Kumar et al. 2008). Additional information will be required to more confidently associate these plateaus with the magnetar model. One of the predictions of the magnetar central engine model is the presence of coherent radio emission from the newly formed magnetar, associated with the plateau phase; this would not be present for the black hole central engine model. Thus, detection of persistent coherent radio emission during the plateau phase of a long GRB would likely rule out a black hole central engine. However, it remains unclear if this emission is able to escape from the local and galactic environment surrounding the GRB location (e.g. Macquart 2007; Lyubarsky 2008; Zhang 2014). There are three key coherent emission models to test:

- persistent pulsar-like emission from the magnetar engine (e.g. Totani 2013).

- FRBs from the young, highly magnetised, neutron star (e.g. Katz 2016; Lyutikov et al. 2016)
- a single FRB at the end of the plateau phase if the neutron star is too massive to support itself and it collapses to form a black hole (Falcke \& Rezzolla 2014; Zhang 2014).

In November 2017, the LOw Frequency ARray (LOFAR; van Haarlem et al. 2013) completed implementing a new rapid response mode, with observations using the full Dutch array starting within 5 minutes of receiving an alert $^{1}$. Although this response time is slower than that of the MWA ( $\sim 30$ seconds), it is sufficiently fast to study the plateau phase and highly dispersed events. By utilising the full Dutch array, a large bandwidth and a two hour observation, we can attain the sensitivity required to deeply probe for emission during the plateau phase. We successfully requested a number of rapid response triggers on GRBs detected by the Niel Gehrels Swift Observatory (hereafter referred to as Swift; Gehrels et al. 2004) and, on 2018 July 6, we successfully completed our first, fully automated, rapid response trigger on GRB 180706A (Stamatikos et al. 2018).

This paper will describe the constraints we can make using the LOFAR observations of GRB 180706A. In Section 2, we describe the observations of GRB 180706A obtained, using Swift and the rapid response mode of LOFAR, and outline our analysis of these data. In Section 3, we consider general coherent radio properties of the emission that can be constrained, we then compare our observations to theoretical models for coherent radio emission associated with both the relativistic jet and the central engine.

\section{OBSERVATIONS OF GRB 180706A}

\subsection{Swift Observations}

GRB 180706A was detected by the Burst Alert Telescope (BAT; Barthelmy et al. 2005) on board the Swift Satellite at 08:24:40 UT on 2018 July 6 (Stamatikos et al. 2018). This GRB was also independently detected by the Fermi Gammaray Burst Monitor (GBM; Meegan et al. 2009; Bissaldi 2018). With a $T_{90}$ duration of $42.7 \pm 8.7$ seconds (15-350 $\mathrm{keV})$, this is a long GRB with a likely collapsar progenitor (Woosley 1993).

Swift automatically slewed to the position of GRB 180706A and X-ray Telescope (XRT; Burrows et al. 2005) observations started 87.7 seconds after the trigger (Stamatikos et al. 2018) and a bright and rapidly fading Xray counterpart was observed. At 95 seconds after the trigger, the UV and Optical Telescope on board Swift (UVOT; Roming et al. 2005) started conducting observations and detected the optical counterpart at $19.88 \pm 0.34$ mag (white filter; Oates \& Stamatikos 2018a). This optical counterpart was also detected by other facilities and shown to be fading (Watson et al. 2018; Volnova et al. 2018; Ulaczyk et al. 2018).

The gamma-ray data from the BAT and the unabsorbed X-ray data from XRT were obtained using the Swift Burst Analyser (Evans et al. 2010) in the $0.3-10 \mathrm{keV}$ energy band. The light curve is characterised by three bright peaks followed by a steep decay and a plateau phase. At $\sim 10^{4} \mathrm{~s}$,

1 http://www.astron.nl/radio-observatory/lofar-systemcapabilities/responsive-telescope/responsive-telescope 


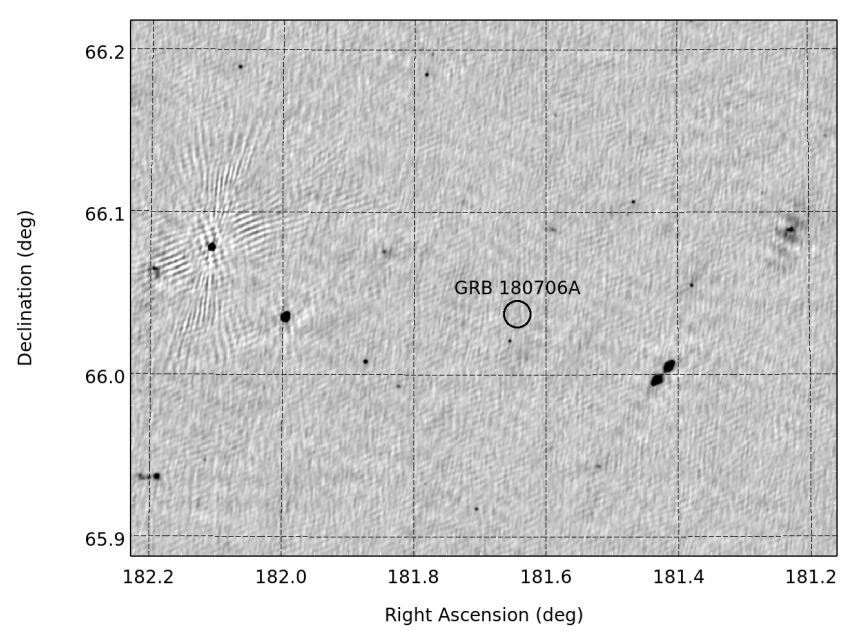

Figure 1. This 2 hour integration LOFAR image shows the region surrounding GRB 180706A at $144 \mathrm{MHz}$. The circle shows the position of GRB 180706A and there is a $3 \sigma$ upper limit of 1.7 mJy beam ${ }^{-1}$ for the flux density of this event.

the plateau turns over to a power law decay phase. Unfortunately, no redshift was obtained for GRB 180706A, though we infer an upper limit of $z \lesssim 2$ from the lowest wavelength in which the optical afterglow was detected (Oates \& Stamatikos 2018b).

\subsection{LOFAR Observations}

We received the VOEvent (Williams \& Seaman 2006) published by Swift via the 4 PI SKY BROKER (using Comet; Staley \& Fender 2016; Swinbank 2014) and, after checking the GRB met our criteria (including source elevation and calibrator availability), automatically triggered observations by sending an XML observing request to the LOFAR system. Our trigger was successfully scheduled and a two hour observation started at 08:29:14 UTC on 2018 July 6, approximately 4.5 minutes after the GRB. We used the BAT position as the pointing centre of our observation. Immediately following this observation, we automatically completed a 2 minute observation of the calibrator source, 3C295.

Our observational set-up was chosen to closely match that of the LOFAR Two-meter Sky Survey (LoTSS; Shimwell et al. 2018); this choice allows us to utilise the deep 8-hour survey images, when available, for comparison to our observations on the event of a detection of radio emission at the GRB location. The observations were completed using the LOFAR High Band Antennas (HBA), with a frequency range of $120-168 \mathrm{MHz}$ and a central frequency of $144 \mathrm{MHz}$, covered by 244 sub-bands each with a bandwidth of 195.3 $\mathrm{kHz}$. We used only the Dutch LOFAR stations, 22 core stations and 11 remote stations, covering projected baselines of $24 \mathrm{~m}$ to $60 \mathrm{~km}$. The data were recorded using a time-step of 1 second and 64 channels per sub-band. We used the standard methods to pre-process our observations (van Haarlem et al. 2013), keeping the 1 second time-step in the archived observations but averaging to 16 frequency channels per sub-band to reduce data volume.

\subsubsection{Calibration}

We used PREFACTOR ${ }^{2}$ to calibrate the target data, following and adapting the strategy used in van Weeren et al. (2016) accordingly. This processing includes flagging of baselines with excess radio frequency interference (RFI) using the AOFLAGGER (Offringa et al. 2010, 2012). Additionally, baselines containing the stations CS021 and CS026 were flagged due to increased noise from these stations. Finally, contributions from the brightest radio sources in the sky, referred to as the A-team, were flagged. For this analysis, the calibrator and target data were both averaged in time to $10 \mathrm{~s}$ and $48.82 \mathrm{KHz}$ (4 channels per subband). Diagonal gain solutions were obtained toward the calibrator source, 3C295, using the model defined by Scaife \& Heald (2012).

The calibrator gain solutions were transferred to the target visibility data. The target subbands were combined in groups of 27, resulting in combined datasets of $5.272 \mathrm{MHz}$. We obtained a sky model of the target field using the global sky model developed by Scheers (2011) and the TIFR GMRT Sky Survey at $150 \mathrm{MHz}$ (TGSS; Intema et al. 2017) ${ }^{3}$. Phase calibration of these datasets was carried out on a $10 \mathrm{~s}$ time scale, using this skymap of the target field.

\subsubsection{Imaging}

We used the LoTSS pipeline ${ }^{4}$ in the manner described by Shimwell et al. (2018) to carry out direction-dependent selfcalibration and imaging of the full 2-hour observation. The final product was a direction-dependent calibrated image of the full dataset, made using the direction-dependent imager DDFACET (Tasse et al. 2018), with a central frequency of 144 $\mathrm{MHz}$ and a bandwidth of $48 \mathrm{MHz}$, using the settings outlined in Shimwell et al. (2018). We show the region surrounding GRB 180706A in Figure 1. The image RMS at GRB location (30 arcsec radius) is $0.58 \mathrm{mJy}^{\text {beam }}{ }^{-1}$, corresponding to a $3 \sigma$ upper limit of 1.7 mJy beam $^{-1}$. Using the Python Source Extractor (PYSE; Carbone et al. 2018) we also conduct a forced source extraction at the position of the GRB holding the shape and size of the Gaussian shape fitted fixed to the restoring beam shape. We measure a peak flux density of $1.1 \pm 0.9 \mathrm{mJy} \mathrm{beam}^{-1}$ (the uncertainty on this value is as measured by PYSE and hence excluding the image RMS noise Carbone et al. 2018).

In addition to the 2 hour integrated image, we also imaged the target data on four snapshot time scales to search for short duration coherent radio emission. We used the sources modelled in the 2 hour integrated image, during the direction dependent and self calibration stages, to subtract them from the target visibilities. This subtraction enables us to probe deeper at the location of the GRB.

We created Stokes I images of these source-subtracted visibilities using WSCLEAN (Offringa et al. 2014) ${ }^{5}$ with Briggs weighting, a pixel scale of 10 arcseconds and baselines up to $12 \mathrm{~km}$. Using the intervals-out option in WSCLEAN, we created snapshot images of durations 30 seconds, 2 minutes, 5 minutes and 10 minutes (the motivation for this range of

2 https://github.com/lofar-astron/prefactor

3 TGSS catalogue: http://tgssadr.strw.leidenuniv.nl/doku.php

4 https://github.com/mhardcastle/ddf-pipeline

5 http://wsclean.sourceforge.net 


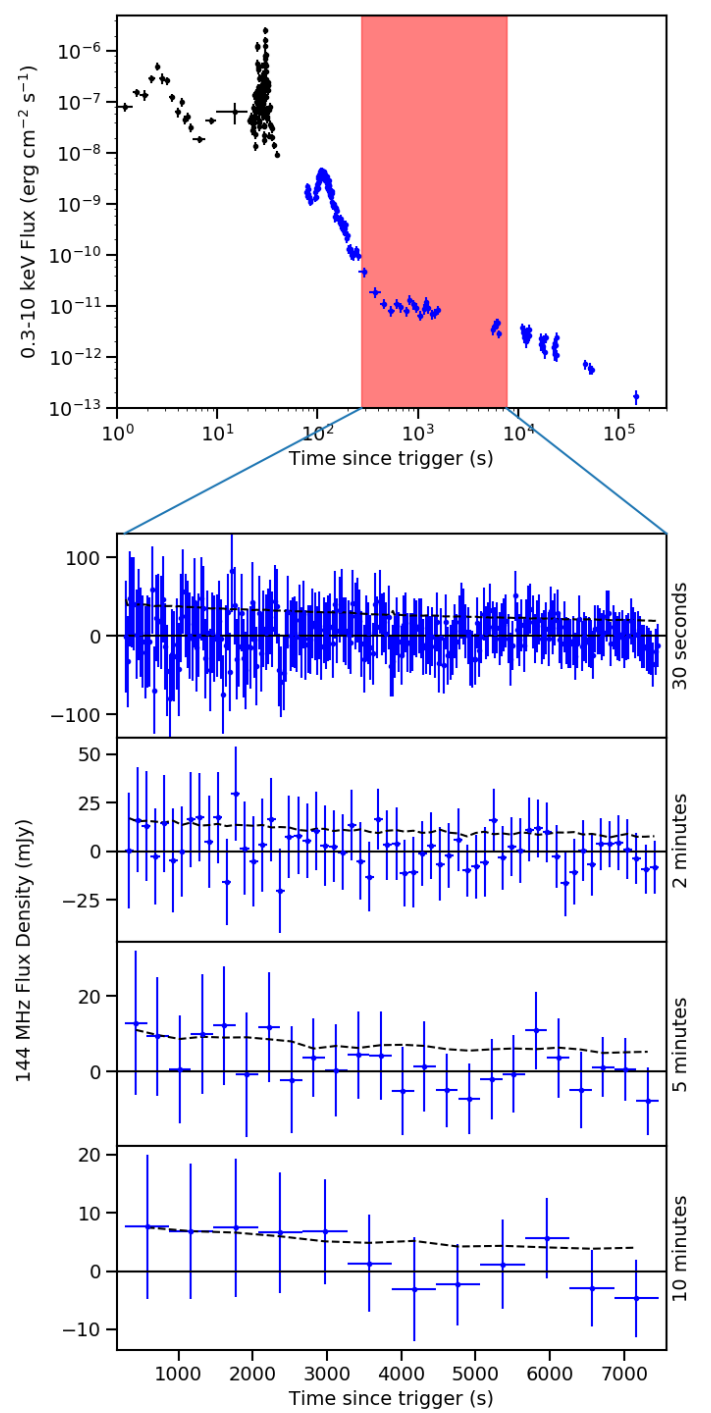

Figure 2. In the top panel of this figure we show the $0.3-10 \mathrm{keV}$ flux light curve of GRB 180706A. The black data points were obtained by the BAT (extrapolated to $0.3-10 \mathrm{keV}$ ) and the blue data points are from the XRT (observed at $0.3-10 \mathrm{keV}$ ). The red shaded region illustrates the time of the LOFAR observation. In the bottom panel, we plot the $144 \mathrm{MHz}$ radio flux density observations as a function of time since the GRB trigger obtained by LOFAR. We show four different snapshot time scales: 30 seconds, 2 minutes, 5 minutes and 10 minutes. The solid black line shows a flux density of $0 \mathrm{mJy}$ and the black dashed lines in each of the LOFAR light curves are the rms noise of the images; measured from the inner $\frac{1}{8}$ th of the image.

time scales is outlined in Section 3.2). The images have a typical angular resolution of $\sim 30$ arcsec.

\begin{tabular}{cc}
\hline $\begin{array}{c}\text { Time scale } \\
\text { (minutes) }\end{array}$ & $\begin{array}{c}\text { rms noise } \\
\left(\text { mJy beam }^{-1}\right)\end{array}$ \\
\hline 0.5 & $28 \pm 6$ \\
2 & $11 \pm 2$ \\
5 & $7 \pm 2$ \\
10 & $5 \pm 1$ \\
\hline
\end{tabular}

Table 1. The average rms noise for the images from each time scale with the $1 \sigma$ standard deviation.

\subsubsection{Image analysis using the LOFAR Transients Pipeline}

The images created were input into the LOFAR Transients Pipeline (TraP; Swinbank et al. 2015) ${ }^{6}$; a pipeline designed to automatically process radio images to search for transient and variable sources. This pipeline measures the rms (root mean square) noise in the inner $\frac{1}{8}$ th of the images. In Table 1 , we give the typical $\mathrm{rms}$ noise for the different imaging time scales.

We use the monitoring list capability of TrAP. Inputting the GRB location into the monitoring list option causes TrAP to measure the flux density at the location of the GRB ${ }^{7}$.

In Figure 2, we show the observations obtained by Swift and the light curves produced by TRAP for each of the different time scales of snapshot images (the integration time of each image is shown by the horizontal error bars), with the image rms over plotted with the black lines. As can be seen from this Figure, the flux densities at the GRB location are consistent with the noise in the inner $\frac{1}{8}$ th of the images (n.b. in some images the local flux measurement, represented by the blue data points, can be lower than the image rms due to the local $\mathrm{rms}$ being slightly lower than the image rms). We note from these snapshot images that the observed flux density at the location of the GRB is marginally positive but consistent with zero within the uncertainties. Therefore, no coherent emission was detected from GRB 180706A in this analysis.

\section{THEORETICAL INTERPRETATION OF OBSERVATIONS}

In the previous Section, we showed that we did not detect any coherent radio emission from GRB 180706A to deep limits. In this Section, we compare our non-detection to the theoretical models that predicted emission during the plateau phase.

\subsection{Propagation considerations}

First, we consider if coherent radio emission is able to escape the dense region surrounding the central engine and the surrounding host galaxy. Macquart (2007) showed that induced Compton and Raman scattering can significantly impede the passage of the coherent radio emission. They

${ }^{6}$ https://github.com/transientskp/tkp

7 For further information see Swinbank et al. (2015) and the TRAP documentation; http://docs.transientskp.org 
show that the emission can only escape if it is ultra relativistic $\left(\Gamma \gtrsim 10^{3} \frac{D}{100 \mathrm{Mpc}}\right)$ or emitted into a very narrow cone. To date, only lower limits on the GRB Lorentz factors have been observed (e.g. ?)Ackermann2010,zhao2011 and they are known to be narrowly beamed although precise jet opening angles are still being constrained; it is therefore unknown if this is sufficient for the emission to escape. Zhang (2014) showed that the emission is likely to escape in the case of short GRBs. Therefore, it is unclear if the emission can escape but, if it does, it would place constraints on the Lorentz factor and the jet opening angle.

Once the coherent radio emission has escaped the immediate surroundings of the GRB, it still needs to travel through the host galaxy and this can lead to further absorption and scattering. Long GRBs are typically found in dense star formation regions near the centres of their host galaxies, making it likely that there is a large absorption column between the event and the Earth. Using the observed absorption in the X-ray spectrum, we can gain an understanding of the total absorption that the coherent radiation will pass through. The X-ray spectrum of GRB 180706A is best fit by a power law, with a photon index of $2.16_{-0.16}^{+0.17}$, and a total absorption column of $N_{H}=6.7_{-3.3}^{+3.7} \times 10^{20} \mathrm{~cm}^{-2}$ (including the Galactic component from the Milky Way of $6.7 \times 10^{20} \mathrm{~cm}^{-2}$; Willingale et al. 2013). Therefore, there are relatively low levels of absorption between the GRB location and Swift so the GRB likely occurred away from the most dense regions in its host galaxy increasing the likelihood of getting prompt radio emission to be observable once it has escaped from the central engine.

Therefore, while it looks more promising for short GRBs, we think there is a chance that the emission from the central engine would have been able to propagate to the Earth for this long GRB.

\subsection{Constraints on Fast Radio Bursts}

There is evidence that repeating FRBs may be linked to Long GRBs. The host galaxy type of the only localized repeating FRB, FRB 121102, is the preferred environment of Long GRBs (Tendulkar et al. 2017; Marcote et al. 2017; Bassa et al. 2017; Kokubo et al. 2017). This is consistent with evolutionary links between FRBs and long GRBs, and possibly between FRBs and magnetars by extension. In this scenario, FRBs are produced from a young magnetar embedded in a supernova remnant (SNR, e.g. Metzger et al. 2017). Using FRB 121102 as a prototype, Law et al. (2017) estimate the volumetric rate of FRBs and find it to be consistent with the rate of Long GRBs. Nicholl et al. (2017) also reach a similar conclusion, supportive of the Long GRB connection to FRBs. A potential caveat with detecting this type of emission very soon after the Long GRB is that the ejecta surrounding the magnetar may prevent the FRBs from escaping causing them to be be detectable only once the SNR has sufficiently expanded. For instance, Cao et al. (2017) and Metzger et al. (2017) estimate a minimum age of $\sim 10-100$ years for FRB 121102. Indeed, other FRB sources detected in real time have not led to counterpart detections at other frequencies (Petroff et al. 2015; Keane et al. 2016; Ravi et al. 2016; Petroff et al. 2017; Bhandari et al. 2018; Farah et al. 2019). However, in this study, we are searching for coher- ent emission before a SNR has had the time to materialize and assume in the same way as in the previous discussion that the FRB emission can escape. Previous prompt FRB searches following Long GRB detections did not result in a firm detection (Bannister et al. 2012; Palaniswamy et al. 2014).

Another possible way to obtain an FRB is via the collapse of the central engine into a black hole as it becomes too massive to support itself (Falcke \& Rezzolla 2014; Zhang 2014), which would be expected at the end of the plateau phase.

It is unclear whether FRBs are detectable at $144 \mathrm{MHz}$. The lowest frequency at which FRBs have been detected is $400 \mathrm{MHz}$ (the bottom of the CHIME/FRB observing band) (CHIME/FRB Collaboration et al. 2019). However, the fact that some of the FRBs detected at $400 \mathrm{MHz}$ show no or negligible scattering, suggests that FRBs are detectable at lower frequencies. The dearth of FRBs detected at lower frequencies may be due to the intrinsic FRB emission mechanism. More likely, though, is free-free absorption at low frequencies (e.g. Lyutikov et al. 2016; Piro 2016). At cosmological distances, however, the turnover in the rest frame spectrum is Doppler shifted (Rajwade \& Lorimer 2017):

$$
v_{\text {rest }}=v_{\text {observed }}(1+z) \text {. }
$$

Using a rest frame spectral turnover due to free-free absorption of $300 \mathrm{MHz}$ (Lyutikov et al. 2016) and setting $v_{\text {observed }}=120 \mathrm{MHz}$ (the bottom of our observing band), our observations could be sensitive to long GRBs at $z>1.5$. We have assumed in this discussion that the plasma frequency, $v_{p}$ is below $144 \mathrm{MHz}$ and therefore is not a limiting factor (for $v_{p}>144 \mathrm{MHz}$, the electron number density would have to be at least $1.6 \times 10^{4} \mathrm{~cm}^{-3}$ ).

Assuming that the FRB emission is able to escape, we may be able to detect it in our snapshot images. To optimise the chances of detection, here we calculate a range of snapshot durations that probe different minimum flux densities and dispersion measure (DM) regimes. As the snapshot durations are greater than the width of the signal, we can follow the method described by Trott et al. (2013) and estimate the minimum FRB flux densities $S_{\text {FRB,min }}$ that we are sensitive to, using

$$
S_{\mathrm{FRB}, \min }=S_{\mathrm{img}}\left(\frac{\Delta t_{\text {int }}}{w}\right),
$$

where $S_{\text {img }}$ is the sensitivity in one snapshot image, $\Delta t_{\text {int }}$ is the snapshot integration time and $w$ is the intrinsic width of the FRB. For consistency with previous works, we assume that the intrinsic width is $1 \mathrm{~ms}$.

Image noise scales with integration time as

$$
S_{\mathrm{img}} \propto \Delta t_{\mathrm{int}}^{-\frac{1}{2}} .
$$

We apply $S_{\text {img }}=1.7 \mathrm{mJy}$ beam $^{-1}$ of the 2 -hour observation to Equation 3 in order to obtain a scaling relationship for our data, and substitute this into Equation 2 to create the relationship between the minimum FRB flux to which we are sensitive as a function of snapshot duration, shown in Figure 3.

Equation 2 is true only if the duration of the dispersed signal, $\Delta t_{\text {dispersion }}$, is equal to $\Delta t_{\text {int }}$. The dispersion delay of a 


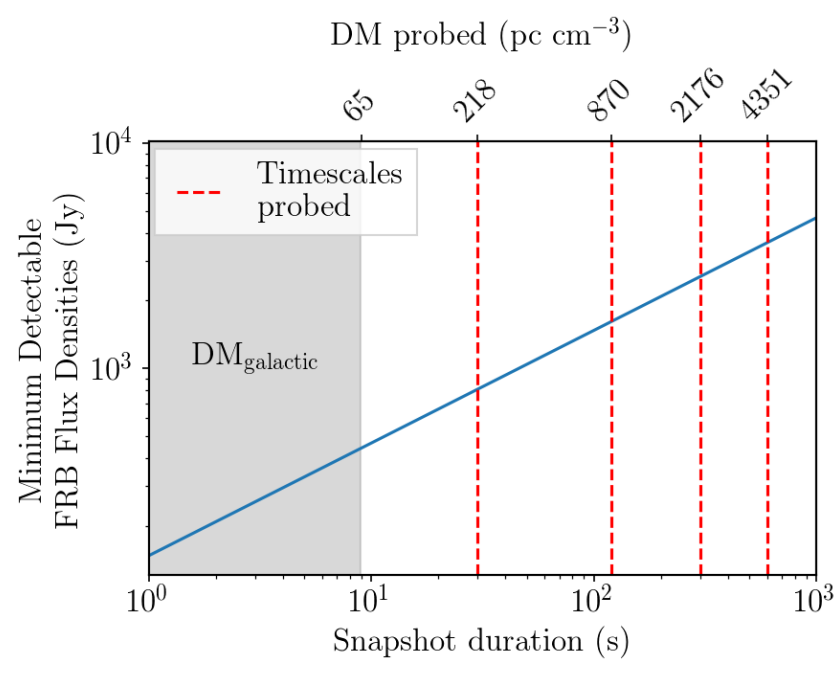

Figure 3. Minimum FRB flux density that can be detected in our 144-MHz LOFAR data as a function of snapshot duration, assuming the duration of the dispersed signal is equivalent to the snapshot duration. The snapshot durations used in this study are marked by red dashed vertical lines. The top $\mathrm{x}$-axis shows the corresponding DM value. The shaded region covers the galactic $\mathrm{DM}$ contribution $\left(\mathrm{DM}_{\text {galactic }}=65 \mathrm{pc} \mathrm{cm}^{-3}\right.$; see Section 3.2) to the total DM along the line of sight of GRB 180706A. This acts as a lower limit in our search parameter space.

radio signal is described as (Lorimer \& Kramer 2005):

$\Delta t_{\text {dispersion }}=4.15 \times 10^{3} \mathrm{MHz}^{2} \mathrm{pc}^{-1} \mathrm{~cm}^{3} \mathrm{~s}\left(\frac{1}{v_{\text {bottom }}^{2}}-\frac{1}{v_{\text {top }}^{2}}\right) \mathrm{DM}$,

where DM is the dispersion measure expressed in $\mathrm{pc} \mathrm{cm}^{-3}$ (i.e. the integrated column density of free electrons along the line of sight), and $v_{\text {bottom }}=120.5 \mathrm{MHz}$ and $v_{\text {top }}=167 \mathrm{MHz}$ are the limits of the observing band. In the top x-axis of Figure 3 , we have substituted $\Delta t_{\text {int }}$ into Equation 4 to visualize the optimal snapshot duration for a given FRB's DM value.

Thus, the choice of snapshot time scales to use is an optimization between DM probed and FRB sensitivity. We can set a minimum snapshot duration of $\sim 9 \mathrm{~s}$, which corresponds to the galactic DM contribution along the line of sight of GRB 180706A, DM $\mathrm{Dalactic}=65 \mathrm{pc} \mathrm{cm}^{-3}$. We use four snapshot time scales in this study: 30 seconds, 2 minutes, 5 minutes, and 10 minutes (as described in Section 2.2.2). Therefore, this experiment is sensitive to FRBs with flux densities in excess of $800 \mathrm{Jy}$ (value extrapolated from Figure 3). Using a reasonable FRB flux density of $1 \mathrm{Jy}$ at $1.4 \mathrm{GHz}$, our experiment sensitivity would require a spectral index $\alpha \lesssim-3$. We note that FRB fluxes at $\sim 1.4 \mathrm{GHz}$ have reached $\sim 150 \mathrm{Jy}$, which would flatten the spectral index required to $\sim-0.8$.

Above we have demonstrated the important role of source DM in searches for coherent emission in radio images. It is difficult to predict the DM of coherent radio emission associated with GRB180706A. The main components of the total $\mathrm{DM}$ are the local burst environment $\left(\mathrm{DM}_{\text {local }}\right)$, the host galaxy $\left(\mathrm{DM}_{\text {host }}\right)$, the intergalactic medium (DM $\left.\mathrm{DGM}_{\mathrm{IGM}}\right)$, the Milky Way's halo $\left(\mathrm{DM}_{\text {halo }}\right)$ and the interstellar medium $\left(\mathrm{DM}_{\mathrm{ISM}}\right.$, i.e. from the Milky Way's disk and spiral arms).
According to the NE2001 model of free electrons in our Galaxy (Cordes \& Lazio 2002), DM ISM should be $35 \pm$ $7 \mathrm{pc} \mathrm{cm}^{-3}$ along the line of sight of GRB180706A. Following Dolag et al. (2015), $\mathrm{DM}_{\text {halo }}$ is $30 \mathrm{pc} \mathrm{cm}^{-3}$. Using an assumed redshift $z=0.2$ allows us to follow the line of thinking from Tendulkar et al. (2017) for FRB 121102, which has $z \approx 0.19$ to estimate a mean DM contribution from the IGM $\mathrm{DM}_{\text {IGM }} \approx 200 \pm 85 \mathrm{pc} \mathrm{cm}^{-3}$. It is difficult to estimate $\mathrm{DM}_{\text {host }}$ without a host galaxy identification, as the value depends on galaxy type and viewing angle, though previous FRB related studies have used $100 \mathrm{pc} \mathrm{cm}^{-3}$ (e.g. Thornton et al. 2013; Xu \& Han 2015; Caleb et al. 2016). As a lower limit at $z=0.2$, we estimate $\mathrm{DM}_{\text {total }} \gtrsim 243 \mathrm{pc} \mathrm{cm}^{-3}$. In the upper limit case of $z \sim 2, \mathrm{DM}_{\mathrm{IGM}}$ dominates over both $\mathrm{DM}_{\text {host }}$ and $\mathrm{DM}_{\text {galactic }}$ with values that can reach $2000 \mathrm{pc} \mathrm{cm}^{-3}$ (Ioka 2003; Inoue 2004). The $\mathrm{DM}_{\text {local }}$ component (i.e. from the SNR) is difficult to estimate but can be significant (Connor et al. 2016), particularly in the first few years of the NS.

Clearly, estimating the source DM beyond the contributions from our Galaxy is difficult and convoluted. Cases where the GRB has an associated host galaxy facilitate constraints on the total DM estimation. A larger population of FRBs should eventually provide insight as to the distribution of free electrons in the IGM. Until then, a more targeted search for coherent emission in radio images can be accomplished through image-plane de-dispersion. However, this is beyond the scope of the paper and will be presented elsewhere. In this study, we have simply chosen timescales that take into account the estimated minimum DM value and that probe parts of the search parameter space (Figure 3) within reason given the uncertainties involved.

\subsection{Constraints on prompt coherent radio emission}

If there were a radio flare emitted from the same region as the gamma-ray emission, it would be reasonable to assume that they could originate from the same emission mechanism. Taking the model by Usov \& Katz (2000), we assume that the gamma-ray and coherent radio emission both originate from magnetic re-connection in strongly magnetized winds within the GRB relativistic jet. In this scenario, the bolometric radio fluence, $\Phi_{r}$, is directly proportional to the bolometric gamma-ray fluence, $\Phi_{\gamma}$, where the power ratio $\langle\delta\rangle$ is given by:

$$
\langle\delta\rangle=\frac{\Phi_{r}}{\Phi_{\gamma}} .
$$

Usov \& Katz (2000) show that this power ratio is roughly equivalent to $\langle\delta\rangle \simeq 0.1 \epsilon_{B}$, where $\epsilon_{B}$ is the proportion of energy contained within the magnetic fields. The bolometric radio fluence is related to the observed radio fluence, $\Phi_{v}$, at an observing frequency, $v$, for frequencies above the peak radio frequency, $v_{\max }$, by:

$$
\Phi_{v}=\frac{\beta-1}{v_{\max }} \Phi_{r}\left(\frac{v}{v_{\max }}\right)^{-\beta},
$$

where $\beta$ is the spectral index. Below $v_{\max }$ there is no observable emission. From Usov \& Katz (2000), for typical parameters of cosmological GRBs,

$$
v_{\max } \simeq[0.5-1] \frac{1}{1+z} \epsilon_{B}^{\frac{1}{2}} \times 10^{6} \mathrm{~Hz} .
$$


By substitution into Equation 5, using the typical value of $\beta=1.6$ (Usov \& Katz 2000) and the observing frequency of $144 \mathrm{MHz}$, we can show

$$
\begin{aligned}
\langle\delta\rangle & =\frac{v^{\beta}}{\beta-1} v_{\max }^{1-\beta} \frac{\Phi_{v}}{\Phi_{\gamma}} \\
\rightarrow\langle\delta\rangle & \simeq[4.7-7.2] \times 10^{9}(1+z)^{0.6} \epsilon_{B}^{-0.3} \frac{\Phi_{v}}{\Phi_{\gamma}}
\end{aligned}
$$

The gamma-ray fluence of GRB 180706A is well constrained, as it was observed by Fermi GBM to be $(3.3 \pm 0.2) \times 10^{-6}$ erg $\mathrm{cm}^{-2}$ in the $10-1000 \mathrm{keV}$ energy band (Bissaldi 2018). We can determine a conservative $3 \sigma 144 \mathrm{MHz}$ radio fluence upper limit, by taking the rms noise in the shortest duration images (multiplying by the duration and 3 to obtain $3 \sigma$ ) from Table 1. The radio fluence limit at $144 \mathrm{MHz}$ in a 30 second snapshot is therefore $\Phi_{v} \leq 840 \pm 180 \mathrm{mJy} \mathrm{s} \lesssim(8.4 \pm$ 1.8) $\times 10^{-18} \mathrm{erg} \mathrm{cm}^{-2} \mathrm{~Hz}^{-1}$. Hence, we find

$$
\langle\delta\rangle \lesssim[0.010-0.024](1+z)^{0.6} \epsilon_{B}^{-0.3}
$$

The redshift is an unknown quantity for GRB 180706A, however we are able to constrain it under the assumption that we would be able to observe coherent radio emission from the prompt emission phase. In addition to the propagation effects within the local environment and host galaxy (outlined in Section 3.1) and as described in 3.2, radio emission is subjected to a frequency dependent delay due to plasma along the line of sight, which is commonly referred to as the dispersion delay. We note that, at low radio frequencies, the dispersion delay for extra galactic events may be several minutes (see also Figure 3). Therefore, if there were prompt radio emission associated with the prompt gammaray emission, the radio emission will arrive after the gammaray emission. Using the following relation from Taylor \& Cordes (1993):

$$
\tau=\frac{\mathrm{DM}}{241 v_{\mathrm{GHz}}^{2}} s
$$

where $\tau$ is the delay between the emission and the radio signal arriving. Given the 4.5 minute delay between the GRB and the start of the LOFAR observations of GRB 180706A, we would be able to search for prompt coherent radio emission for events with a DM of $1350 \mathrm{pc} \mathrm{cm}^{-3}$. Using the approximate relation between $\mathrm{DM}$ and redshift $(D M \sim 1200 z \mathrm{pc}$ $\mathrm{cm}^{-3}$, e.g. Ioka 2003), this corresponds to events at $z \gtrsim 1.1$.

Hence, using the relation $\langle\delta\rangle \lesssim 0.1 \epsilon_{B}$ and assuming a redshift of 1.1, we can use Equation 10 to constrain the maximum value of $\epsilon_{B}$ to be $\epsilon_{B} \lesssim[0.24-0.47]$. This value of $\epsilon_{B}$ is with the expectation for a magnetically dominated GRB jet (e.g. Beniamini \& Piran 2014). Therefore, although we are unable to accurately constrain this model due to the uncertain parameters for GRB $180706 \mathrm{~A}$, we show that we are achieving sufficient sensitivity to either confirm a magnetically dominated jet or to rule this out. Using future rapid response radio observations of GRBs we may be able to determine if the jet is baryon dominated or magnetically dominated and answer one of the outstanding questions in GRBs (e.g. Sironi et al. 2015).

\subsection{Constraints on the magnetar central engine model}

In the magnetar central engine model, there are predictions of persistent coherent radio emission from the magnetar. This is in addition to the possible FRB emission considered in Section 3.2. The persistent emission is typically considered to be from pulsar-type emission (e.g. Totani 2013). As we have deep LOFAR observations during the plateau phase, when the magnetar emission is expected to dominate, we can place constraints on this models. First we model the X-ray light curve to determine the magnetar parameters, then we use these parameters to predict the coherent radio emission expected during our observations.

\subsubsection{Modelling of X-ray light curve}

In the case of GRB 180706A, the redshift is unknown so the luminosity and rest frame duration of the X-ray plateau are subsequently unknown. Therefore, in this analysis we assume a redshift value to calculate and fit the rest frame light curve. We choose $z=0.2$ as an arbitrary reference point and show how the results obtained with this chosen redshift can be scaled to other redshift values. Assuming the spectrum can be described by a single power law (as fitted in the X-ray), the light curve was then converted into a rest frame 1-10000 keV luminosity light curve using a k-correction (Bloom et al. 2001) giving an approximately bolometric light curve shown in Figure 4.

The rest frame light curve was fitted with the magnetar model (Zhang \& Mészáros 2001), using the method described in Rowlinson et al. (2013). The magnetar model is given by:

$$
\begin{aligned}
& B_{15}^{2}=4.2025 M_{1.4}^{2} R_{6}^{-2} L_{0,49}^{-1} T_{\mathrm{em}, 3}^{-2} f, \\
& P_{-3}^{2}=2.05 M_{1.4} R_{6}^{2} L_{0,49}^{-1} T_{\mathrm{em}, 3}^{-1} f,
\end{aligned}
$$

where $B=10^{15} B_{15} \mathrm{G}$ is the magnetic field of the magnetar, $P=10^{-3} P_{-3} \mathrm{~s}$ is the initial spin period of the magnetar, $R=10^{6} R_{6} \mathrm{~cm}$ is the radius of the magnetar, $M=1.4 M_{1.4}$ $\mathrm{M}_{\odot}$ is the mass of the magnetar, $T=10^{3} T_{\mathrm{em}, 3}$ is the plateau duration and $L=10^{49} L_{0,49}$ is the plateau luminosity. Here,

$$
f=\left(\frac{\epsilon}{1-\cos \theta}\right)^{0.5}
$$

is a factor encompassing all the uncertainties in the beaming angle, $\theta$, and the efficiency of conversion of the spin energy into X-rays, $\epsilon$. When $f=1$ the system is assumed to emit isotropically with 100 per cent efficiency. Using the observational constraints from Rowlinson et al. (2014), it can be shown that $f \sim 3.45$ by calculating the average of this ratio for all the combinations of beaming angle and efficiency that produced a $>95 \%$ probability of being able to explain the observed data (c.f. figure 3 in Rowlinson et al. 2014).

As shown in Rowlinson \& Anderson (2019), the magnetic field and spin parameters can be scaled to different redshifts using these relations:

$$
\begin{aligned}
B_{15} & \propto \frac{(1+z)}{D_{L}}, \\
P_{-3} & \propto \frac{(1+z)^{\frac{1}{2}}}{D_{L}} .
\end{aligned}
$$




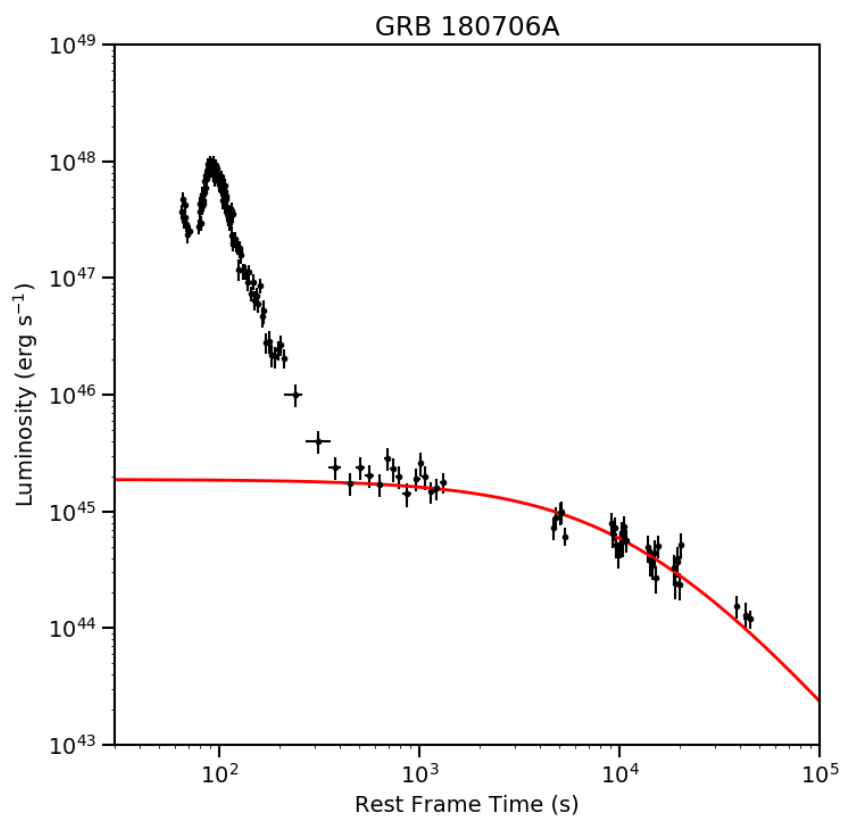

Figure 4. This figure shows the rest frame X-ray light curve (black data points, XRT data only), assuming a redshift of 0.2 . The red line shows the magnetar model fit obtained, corresponding to a magnetic field of $B=11.83_{-0.71}^{+0.76} f \times 10^{15} \mathrm{G}$ and spin period of $P=29.37_{-0.64}^{+0.68} f \mathrm{~ms}$.

A cosmology calculator can then be used to convert the required redshift to a luminosity distance $\left(D_{L}\right)^{8}$.

We find that the plateau, and subsequent decay phase, can be fitted by a newly-formed stable magnetar with a magnetic field of $11.83_{-0.71}^{+0.76} f \times 10^{15} \mathrm{G}$ and spin period of $29.37_{-0.64}^{+0.68} f \mathrm{~ms}$ assuming a redshift of 0.2 . This model is plotted in Figure 4.

\subsubsection{Pulsar like emission}

In the emission model proposed by Totani (2013) (see also Pshirkov \& Postnov 2010), coherent emission is expected to occur via magnetic braking (dipole spin-down) of a newly formed magnetar. Totani (2013) assumed that this emission is comparable to that of known pulsars and predict that this emission can be described by:

$$
F_{v} \simeq 8 \times 10^{7} v_{\text {obs }}^{-1} \epsilon_{r} D_{\text {lum }}^{-2} B_{15}^{2} R_{6}^{6} P_{-3}^{-4} \mathrm{Jy}
$$

where $D_{\text {lum }}$ is the luminosity distance in Gpc, $v_{\text {obs }}$ is the frequency in $\mathrm{MHz}, B_{15}=\frac{B}{10^{15}}, P_{-3}=\frac{P}{10^{-3}}, R_{6}=\frac{R}{10^{6}}, R$ is the neutron star radius in metres, and $\epsilon_{r}$ is the efficiency.

These predictions assume that the pulsar magnetic field axis is highly aligned with the rotation axis of the system to enable the emission to escape via the region that the relativistic jet has cleared. This also ensures that the pulsar emission is directed towards the Earth. As shown in Rowlinson et al. (2017), this assumption is reasonable as the dy- namo mechanism produces a magnetic field along the rotation axis and there is insufficient time for the rotation and magnetic axes to become misaligned.

Finally, although the model proposed by Zhang \& Mészáros (2001) assumes that the newly born magnetar emits via dipole radiation, this is not necessarily the case as the magnetic fields may initially be in a different orientation (e.g. quadrupolar). This means the assumption made by Totani (2013), that the newly born magnetar behaves like the known pulsar population, may be unreasonable. Recently, Lasky et al. (2017) investigated this assumption by modelling the late-time decay slope following the plateau phase. They found that the braking indices are consistent with the known pulsar population and have the first detection of a braking index of 3 , which is the value expected for pure dipole radiation. Therefore, it is likely reasonable to assume that these new-born neutron stars are spinning down in a similar manner to known pulsars.

In this analysis, we have assumed a redshift of $z=0.2$ for GRB 180706A to allow us to predict the expected flux density of the radio emission at that redshift. However, as shown in Rowlinson \& Anderson (2019), the predicted radio flux density is directly proportional to the observed X-ray fluxes because they originate from the same emission process. Hence, the predicted radio flux density for this event is independent of the actual redshift of the event.

The efficiency is the remaining unknown quantity in this analysis but, given that the emission is believed to be the same as for pulsars, we use the pulsar value of $10^{-4}$ and illustrate how the predictions vary for a range of values in Figure 5. As the plateau phase observed in GRB 180706A fits the magnetar central engine model, our LOFAR observations for the entire duration of the plateau phase are ideal to test for this emission. The plateau has a long duration, so we can assume that we are in a non-dispersed regime and thus the predicted flux densities are equivalent to the observed values. By using the values fitted for GRB 180706A in Section 3.4.1, an assumed distance of $987 \mathrm{Mpc}^{9}$, the midfrequency of our LOFAR observations (144 MHz), and a 10 $\mathrm{km}$ neutron star radius, we find a predicted flux density of

$$
F_{\nu} \simeq 10.7_{-2.1}^{+2.6} f^{-2} \mathrm{mJy}
$$

By assuming isotropic emission and 100 per cent X-ray efficiency, we can predict an upper limit of the flux density from a pulsar system to be $\sim 10 \mathrm{mJy}$. When the beaming and efficiency factor is assumed to be $\mathrm{f}=3.45$ (using the analysis of Rowlinson et al. 2014), the predicted flux drops to $\sim 1 \mathrm{mJy}$. Our limits are three orders of magnitude deeper than the previous best obtained by the MWA (Kaplan et al. 2015). Assuming that the newly formed magnetar is emitting consistently with the known pulsar population and that the emission can escape the system, the emission would have been likely, or close to, detectable in our observations if its beaming and efficiency properties are consistent with the known GRB magnetar population (Rowlinson et al. 2014). However, if this system were less efficient and/or more highly beamed than the standard GRB magnetar population, the

\footnotetext{
8 e.g.

the

cosmology

calculator

http://www.astro.ucla.edu/ wright/CosmoCalc.html (Wright 2006)
}

9 corresponding to a redshift of 0.2 and using the cosmology calculator http://www.astro.ucla.edu/ wright/CosmoCalc.html (Wright 2006) 


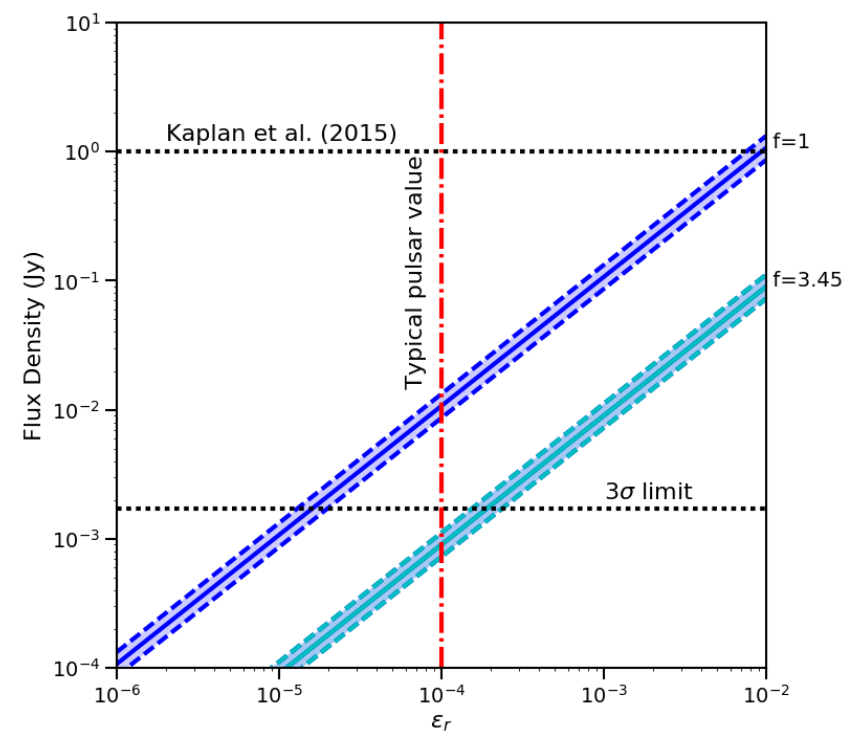

Figure 5. This figure shows the predicted flux density at 144 $\mathrm{MHz}$ as a function of the efficiency of converting the rotational energy into observable radio emission. The red dash-dotted line shows the typical efficiencies for observed pulsars in our own Galaxy. The lower black dotted line shows the $3 \sigma$ upper limit at the position of the GRB in the 2 hour integrated image. The upper black dotted line shows the previous best limits for this emission from Kaplan et al. (2015) for a similar event. The blue line, dashed lines and shaded region illustrates the emission predicted using the parameters obtained for the magnetar from the X-ray plateau assuming $100 \%$ efficiency and isotropic emission $(\mathrm{f}=1)$. The equivalent region in cyan represents the more likely scenario with some combination of beaming and reduced efficiency, corresponding to $\mathrm{f}=3.45$.

emission would not be detectable in our observations. Further deep observations of more GRBs with a plateau phase will be required to rule this scenario out.

\section{CONCLUSIONS}

In this Paper, we have presented the LOFAR rapid response observations of GRB 180706A, starting at 4.5 minutes of the trigger, searching for coherent low frequency radio emission from the central engine. A detection of coherent radio emission would be strong supporting evidence for the Poynting flux dominated jet or the magnetar central engine model. The X-ray data of GRB 180706A are shown to fit the magnetar model, making it a good candidate to search for this emission.

No emission was detected at the location of GRB $180706 \mathrm{~A}$ in the full 2 hour integrated image. Neither was emission detected in the short duration snapshot images, which were targeting FRB like emission. We note that the snapshot images were sensitive to particular DM values; an image plane de-dispersion strategy (such as that conducted by Tingay et al. 2013) will be required to conduct a deeper search. Future work will include a development of an image plane de-dispersion strategy for LOFAR. Additionally, we plan to introduce commensal imaging and beamformed observations into the LOFAR rapid response mode, enabling us to conduct a standard FRB search.

Due to the 4.5 minute response time and the unknown redshift of GRB 180706A, we are unable to constrain the presence of coherent radio emission associated with a Poynting flux dominated jet. However, we demonstrate that LOFAR is attaining the radio sensitivity required to constrain this model with future GRBs.

The non detection of coherent radio emission associated with the X-ray plateau phase currently does not rule out the magnetar central engine model. This is due to a number of reasons:

- The redshift of GRB 180706A is unknown, hence it may be too distant for us to detect FRB like emission.

- There remains significant uncertainty in the coherent emission models, ranging from efficiency factors to the spectrum of the emission.

- Although the X-ray spectrum implies that GRB 180706A may have occurred in a reasonably low density environment, long GRBs are typically expected to be found in high density environments and hence the coherent emission may not be able to escape.

In order to confidently rule out or detect the predicted coherent radio emission, we need multiple rapid response observations of GRBs with radio telescopes of comparable (or better) sensitivity to LOFAR.

\section{ACKNOWLEDGEMENTS}

We thank the LOFAR Radio Observatory for implementing the new rapid response mode and for supporting our observations. MJK, AS and RAMJW acknowledge funding from the ERC Advanced Investigator grant no. 247295. WLW and MJH acknowledge support from the UK Science and Technology Facilities Council [ST/M001008/1]

This paper is based (in part) on data obtained with the International LOFAR Telescope (ILT) under project code LC10_012. LOFAR (van Haarlem et al. 2013) is the Low Frequency Array designed and constructed by ASTRON. It has observing, data processing, and data storage facilities in several countries, that are owned by various parties (each with their own funding sources), and that are collectively operated by the ILT foundation under a joint scientific policy. The ILT resources have benefited from the following recent major funding sources: CNRS-INSU, Observatoire de Paris and Université d'Orlèans, France; BMBF, MIWF-NRW, MPG, Germany; Science Foundation Ireland (SFI), Department of Business, Enterprise and Innovation (DBEI), Ireland; NWO, The Netherlands; The Science and Technology Facilities Council, UK; Ministry of Science and Higher Education, Poland.

This work made use of data supplied by the UK Swift Science Data Centre at the University of Leicester and the Swift satellite. Swift, launched in November 2004, is a NASA mission in partnership with the Italian Space Agency and the UK Space Agency. Swift is managed by NASA Goddard. Penn State University controls science and flight operations from the Mission Operations Center in University 
Park, Pennsylvania. Los Alamos National Laboratory provides gamma-ray imaging analysis.

\section{REFERENCES}

Abbott B. P., et al., 2017, ApJ, 848, L13

Anderson M. M., et al., 2018, ApJ, 864, 22

Balsano R. J., et al., 1998, in Meegan C. A., Preece R. D., Koshut T. M., eds, American Institute of Physics Conference Series Vol. 428, Gamma-Ray Bursts, 4th Hunstville Symposium. pp 585-589, doi:10.1063/1.55382

Bannister K. W., Murphy T., Gaensler B. M., Reynolds J. E., 2012, ApJ, 757, 38

Barthelmy S. D., et al., 2005, Space Sci. Rev., 120, 143

Bassa C. G., et al., 2017, ApJ, 843, L8

Bastian T. S., Benz A. O., Gary D. E., 1998, ARA\&A, 36, 131

Beniamini P., Piran T., 2014, MNRAS, 445, 3892

Bernardini M. G., Margutti R., Mao J., Zaninoni E., Chincarini G., 2012, A\&A, 539, A3

Bhandari S., et al., 2018, Monthly Notices of the Royal Astronomical Society, 475, 1427

Bissaldi E., 2018, GRB Coordinates Network, 22919, 1

Bloom J. S., Frail D. A., Sari R., 2001, AJ, 121, 2879

Burrows D. N., et al., 2005, Space Sci. Rev., 120, 165

CHIME/FRB Collaboration et al., 2019, Nature, 566, 230

Caleb M., Flynn C., Bailes M., Barr E. D., Hunstead R. W., Keane E. F., Ravi V., van Straten W., 2016, MNRAS, 458, 708

Cao X.-F., Yu Y.-W., Dai Z.-G., 2017, ApJ, 839, L20

Carbone D., et al., 2018, Astronomy and Computing, 23, 92

Connor L., Sievers J., Pen U.-L., 2016, MNRAS, 458, L19

Cordes J. M., Lazio T. J. W., 2002, ArXiv Astrophysics e-prints,

Cortiglioni S., Mandolesi N., Morigi G., Ciapi A., Inzani P., Sironi G., 1981, Ap\&SS, 75, 153

Dessenne C. A.-C., et al., 1996, MNRAS, 281, 977

Dolag K., Gaensler B. M., Beck A. M., Beck M. C., 2015, MNRAS, 451,4277

Drenkhahn G., Spruit H. C., 2002, A\&A, 391, 1141

Evans P. A., et al., 2010, A\&A, 519, A102

Falcke H., Rezzolla L., 2014, A\&A, 562, A137

Farah W., et al., 2019, Monthly Notices of the Royal Astronomical Society, 488, 2989

Gehrels N., et al., 2004, ApJ, 611, 1005

Hjorth J., et al., 2003, Nature, 423, 847

Inoue S., 2004, MNRAS, 348, 999

Intema H. T., Jagannathan P., Mooley K. P., Frail D. A., 2017, A\&A, 598, A78

Inzani P., Sironi G., Mandolesi N., Morigi G., 1982, in Lingenfelter R. E., Hudson H. S., Worrall D. M., eds, American Institute of Physics Conference Series Vol. 77, Gamma Ray Transients and Related Astrophysical Phenomena. pp 79-84, doi:10.1063/1.33213

Ioka K., 2003, ApJ, 598, L79

Kaplan D. L., et al., 2015, ApJ, 814, L25

Katz J. I., 2016, ApJ, 826, 226

Keane E. F., et al., 2016, Nature, 530, 453

Kokubo M., et al., 2017, ApJ, 844, 95

Koranyi D. M., Green D. A., Warner P. J., Waldram E. M., Palmer D. M., 1995, MNRAS, 276, L13

Kumar P., Narayan R., Johnson J. L., 2008, MNRAS, 388, 1729

Lasky P. D., Leris C., Rowlinson A., Glampedakis K., 2017, ApJ, 843, L1

Law C. J., et al., 2017, ApJ, 850, 76

Lorimer D. R., Kramer M., 2005, Handbook of pulsar astronomy. Vol. 4, Cambridge University Press

Lyubarsky Y., 2008, ApJ, 682, 1443

Lyutikov M., Burzawa L., Popov S. B., 2016, MNRAS, 462, 941
Macquart J.-P., 2007, ApJ, 658, L1

Marcote B., et al., 2017, ApJ, 834, L8

Meegan C., et al., 2009, ApJ, 702, 791

Metzger B. D., Giannios D., Thompson T. A., Bucciantini N., Quataert E., 2011, MNRAS, 413, 2031

Metzger B. D., Berger E., Margalit B., 2017, ApJ, 841, 14

Mirzoyan R., et al., 2019, GRB Coordinates Network, 23701, 1

Nicholl M., Williams P. K. G., Berger E., Villar V. A., Alexander K. D., Eftekhari T., Metzger B. D., 2017, ApJ, 843, 84

Oates S. R., Stamatikos M., 2018a, GRB Coordinates Network, Circular Service, No. 22922, \#1 (2018/July-0), 22922

Oates S. R., Stamatikos M., 2018b, GRB Coordinates Network, Circular Service, No. 22922, \#1 (2018/July-0), 22922

Offringa A. R., de Bruyn A. G., Biehl M., Zaroubi S., Bernardi G., Pandey V. N., 2010, MNRAS, 405, 155

Offringa A. R., van de Gronde J. J., Roerdink J. B. T. M., 2012, A\&A, 539, A95

Offringa A. R., McKinley B., Hurley-Walker et al., 2014, MNRAS, 444,606

Palaniswamy D., Wayth R. B., Trott C. M., McCallum J. N., Tingay S. J., Reynolds C., 2014, ApJ, 790, 63

Petroff E., et al., 2015, MNRAS, 447, 246

Petroff E., et al., 2017, MNRAS, 469, 4465

Piro A. L., 2016, ApJ, 824, L32

Pshirkov M. S., Postnov K. A., 2010, Ap\&SS, 330, 13

Rajwade K. M., Lorimer D. R., 2017, MNRAS, 465, 2286

Ravi V., et al., 2016, Science, 354, 1249

Roming P. W. A., et al., 2005, Space Sci. Rev., 120, 95

Rowlinson A., Anderson G. E., 2019, arXiv e-prints, p. arXiv:1905.02509

Rowlinson A., O'Brien P. T., Metzger B. D., Tanvir N. R., Levan A. J., 2013, MNRAS, 430, 1061

Rowlinson A., Gompertz B. P., Dainotti M., O'Brien P. T., Wijers R. A. M. J., van der Horst A. J., 2014, MNRAS, 443, 1779

Rowlinson A., Patruno A., O'Brien P. T., 2017, MNRAS, 472, 1152

Scaife A. M. M., Heald G. H., 2012, MNRAS, 423, L30

Scheers L. H. A., 2011, PhD thesis, University of Amsterdam

Shimwell T. W., et al., 2018, arXiv e-prints,

Sironi L., Petropoulou M., Giannios D., 2015, MNRAS, 450, 183

Staley T. D., Fender R., 2016, preprint, (arXiv:1606.03735)

Stamatikos M., et al., 2018, GRB Coordinates Network, Circular Service, No. 22909, \#1 (2018/July-0), 22909

Swinbank J., 2014, Astronomy and Computing, 7, 12

Swinbank J. D., et al., 2015, Astronomy and Computing, 11, 25

Tasse C., et al., 2018, A\&A, 611, A87

Taylor J. H., Cordes J. M., 1993, ApJ, 411, 674

Taylor G. B., et al., 2012, Journal of Astronomical Instrumentation, 1, 1250004

Tendulkar S. P., et al., 2017, ApJ, 834, L7

Thornton D., et al., 2013, Science, 341, 53

Tingay S. J., et al., 2013, Publ. Astron. Soc. Australia, 30, e007

Totani T., 2013, Publications of the Astronomical Society of Japan, 65, L12

Trott C. M., Tingay S. J., Wayth R. B., 2013, ApJ, 776, L16

Ulaczyk K., et al., 2018, GRB Coordinates Network, Circular Service, No. 22924, \#1 (2018/July-0), 22924

Usov V. V., Katz J. I., 2000, A\&A, 364, 655

Volnova A., Reva I., Mazaeva E., Pozanenko A., Kusakin A., Krugov M., 2018, GRB Coordinates Network, Circular Service, No. 22923, \#1 (2018/July-0), 22923

Watson A. M., Butler N., Becerra R. L., Roman-Zuniga C., Lee W. H., 2018, GRB Coordinates Network, Circular Service, No. 22910, \#1 (2018/July-0), 22910

Williams R. D., Seaman R., 2006, in Gabriel C., Arviset C., Ponz D., Enrique S., eds, Astronomical Society of the Pacific Conference Series Vol. 351, Astronomical Data Analysis Software and Systems XV. p. 637 
Willingale R., Starling R. L. C., Beardmore A. P., Tanvir N. R., O'Brien P. T., 2013, MNRAS, 431, 394

Woosley S. E., 1993, ApJ, 405, 273

Woosley S. E., Bloom J. S., 2006, ARA\&A, 44, 507

Wright E. L., 2006, PASP, 118, 1711

Xu J., Han J. L., 2015, Research in Astronomy and Astrophysics, 15,1629

Zhang B., 2014, ApJ, 780, L21

Zhang B., Mészáros P., 2001, ApJ, 552, L35

van Haarlem M. P., et al., 2013, A\&A, 556, A2

van Weeren R. J., et al., 2016, ApJS, 223, 2

This paper has been typeset from a $\mathrm{T}_{\mathrm{E}} \mathrm{X} / \mathrm{LAT}_{\mathrm{E}} \mathrm{X}$ file prepared by the author. 